\title{
SALT Observations of Hot Subdwarfs and Other Evolved Stars
}

\section{Simon Jeffery*}

Armagh Observatory, College Hill, Armagh BT61 9DG, N.Ireland

E-mail: csjearm.ac.uk

SALT has been used to obtain a significant number of observations of hot subdwarfs and hydrogen-deficient stars. The greatest challenge is posed by the helium-rich subdwarfs, of which many have exotic surface compositions and for which an evolutionary origin is urgently sought. A second strand has been the study of pulsating subdwarf B stars, with emphasis on the detection of surface motion in an effort to use geometric methods to measure the stellar radius. Other hot evolved stars including the hydrogen-deficient binary DY Cen have also been observed. We report on progress.

SALT Science Conference 2015 -SSC2015-

1-5 June, 2015

Stellenbosch Institute of Advanced Study, South Africa

\footnotetext{
* Speaker.
} 


\section{Introduction}

The processes of destruction which dismantle stars as they age are just as important as those associated with star formation for understanding the evolution of the cosmos. They are clearly a function of mass, composition and duplicity, but also involve a lot of physics which needs to be better understoood. The outputs lead to the final mass function, the mass and composition of reprocessed material, rates and types of supernovae, the compact-object population, doubledegenerate mergers, and much more. They can be explored by observing highly-evolved stars using pulsations, binaries and surface chemistries. The diversity of the hot star zoo tells us that the ways to break a star far outnumber the ways to make one, and helps us explore diverse phenomena such as the formation of common-envelopes around close binary stars, the opacity of stellar material and the physics of radiatively-driven diffusion.

Armagh Observatory has been using the Southern African Large Telescope (SALT) in studies of hot subdwarfs, both normal $(\S 2)$ and helium-rich $(\S 3)$, in order to explore their pulsation, binary properties and their chemically peculiar surfaces. A particular goal has been to obtain highprecision spectroscopy of pulsating hot subdwarfs and of a related object, the pulsating extreme helium star BX Cir (§ 4). Opportunities to explore the orbits of other hydrogen-deficient systems have also been pursued $(\S 5)$.

\section{Normal sdB stars}

In terms of internal structure, normal subdwarf B (sdB) stars may be thought of as extreme horizontal-branch stars; that is, they are core helium-burning stars of approximately half a solar mass with a very low-mass hydrogen envelope [1]. They have diverse origins, being found mostly in binaries ranging from short-period systems containing $M$ dwarfs or white dwarfs, to long-period systems containing $\mathrm{F}$ and $\mathrm{G}$ dwarfs, and also sometimes found singly [2]. Their surfaces are helium-poor, gravitational settling and radiative levitation causes the helium to sink or, equivalently, the hydrogen to float [3]. Amongst other elements, those with atomic number $Z<20$ generally show sub-solar abundances (except for nitrogen), whilst those with $Z>20$ show super-solar abundances (except for iron) [4]. Again, these patterns can be explained in terms of radiatively-driven diffusion. A crucial aid to the interpretation of these stars is the presence, in some cases, of multi-periodic p- and g-mode pulsations, with periods in the range of 100-600 s [5] and 3000-6000 s respectively [6].

These pulsations provide data for asteroseismological investigations of the internal structure [7]. Potentially they can also provide data for more direct measurements of stellar dimensions, specifically the radius. If the total radial displacement $(\delta R)$ and the relative amplitude of the angular diameter variation $(\delta \theta /\langle\theta\rangle)$ can be measured for a given radial mode, then the radius follows from

$$
R=\delta R\langle\theta\rangle / \delta \theta
$$

The former can be deduced by integrating the radial velocity, whilst the latter may be deduced by combining the variation in effective temperature and total flux. Although less straightforward, a similar approach allows the radius to be deduced for low-degree non-radial models. At least two observables are necessary. High-time and high-spectral resolution spectroscopy is required to 
Table 1: Observing log for SALT-RSS investigations of pulsating sdB stars.

\begin{tabular}{llllll} 
Star & Period Range & Date & Grating & $n \times t_{\text {exp }}$ & Accepted \\
PG 2303+019 & $128-145 \mathrm{~s}$ & 2012 Jun 30 & 0900 & $106 \times 10 \mathrm{~s}$ & No \\
& & 2012 Jul 30 & 0900 & $220 \times 10 \mathrm{~s}$ & Yes - colours \\
& & 2012 Oct 13 & 2300 & $185 \times 20 \mathrm{~s}$ & No - velocities \\
& & 2012 Oct 31 & 0900 & $98 \times 10 \mathrm{~s}$ & Yes - colours \\
2M 0415+0154 & $144-149 \mathrm{~s}$ & 2012 Dec 02 & 3000 & $102 \times 10 \mathrm{~s}$ & No \\
& & 2012 Dec 25 & 3000 & $148 \times 10 \mathrm{~s}$ & No \\
& & 2012 Dec 26 & 3000 & $105 \times 10 \mathrm{~s}$ & Yes - noisy \\
V4640 Sgr & \multirow{2}{*}{$137-159 \mathrm{~s}$} & 2013 Apr 23 & 3000 & $180 \times 13 \mathrm{~s}$ & Yes - velocities \\
& & 2013 Apr 25 & 0900 & $342 \times 13 \mathrm{~s}$ & Yes - colours
\end{tabular}

measure radial velocity amplitudes of a few $\mathrm{km} \mathrm{s}^{-1}$ or less, from which $\delta R$ can be established. The amplitudes of light and colour variations are also required in order to establish the variation in $\theta$. Since $\mathrm{p}$-mode pulsations in $\mathrm{sdB}$ stars have short periods, the relatively short observing runs possible with the SALT Robert Stobie Spectrograph (RSS) have been used in attempts to measure both radial velocity and colour variations.

Observations were attempted through 2012/13 for three stars, V4640 Sgr, PG 2303+019 and $2 \mathrm{M} 0415+0154$. These stars were chosen for having a dominant $\mathrm{p}$-mode pulsation $[8,9,10]$. Table 1 shows the number of spectra and exposure time used for each observing run, the range of $p$-mode oscillations known, and whether the observations were accepted. Low- and medium-resolution gratings were used in efforts to obtain the amplitudes of the dominant mode in both colour and radial velocity, respectively. Observing conditions generally conspired against the project. In other cases, the $\mathrm{S} / \mathrm{N}$ was substantially lower than anticipated for an 11-m telescope; efforts to extract meaningful results continue.

\section{Helium-rich sdO/B stars}

Some $90 \%$ of all hot subdwarfs have surfaces where the helium abundance is either normal or, in the case of sdB stars, substantially below normal. However some $10 \%$ have surfaces which are either partially or extremely hydrogen-deficient. For convenience, these can be roughly divided between extreme He-rich $\mathrm{sdO} / \mathrm{B}$ stars with surface helium-to-hydrogen ratios $n_{\mathrm{He}} / n_{\mathrm{H}}>4$ (or fractional helium abundance $n_{\mathrm{He}}>80 \%$ ) and intermediate $\mathrm{He}$-rich sdO/B stars with surface helium-to-hydrogen ratios $4>n_{\mathrm{He}} / n_{\mathrm{H}}>0.25\left(0.8>n_{\mathrm{He}}>0.2 \%\right)$.

The extreme He-rich subdwarfs may be further divided into carbon- and nitrogen-rich subgroups. The majority are apparently single, and thus an origin in a double helium-white dwarf merger is plausible [11]. One, PG $1544+488$, is a double extreme helium subdwarf; a very finely tuned binary precursor is required to establish two helium cores following a common-envelope ejection episode $[12,13]$. One question is whether any other such systems exist?

The intermediate He-rich subdwarfs are considerably more diverse. CD-20 1123 is a known binary [14]. More extraordinarily, three have quite extreme (4 dex) overabundances of exotic el- 
ements (zirconium, germanium, yttrium, strontium, and lead) on their surfaces [15, 14], whilst another three show similarly large excesses of iron-group elements [16].

SALT has been used to explore both groups of He-rich subdwarfs in an effort to (1) identify and characterize additional binaries and (2) pursue abundance studies of intermediate He-rich sdBs.

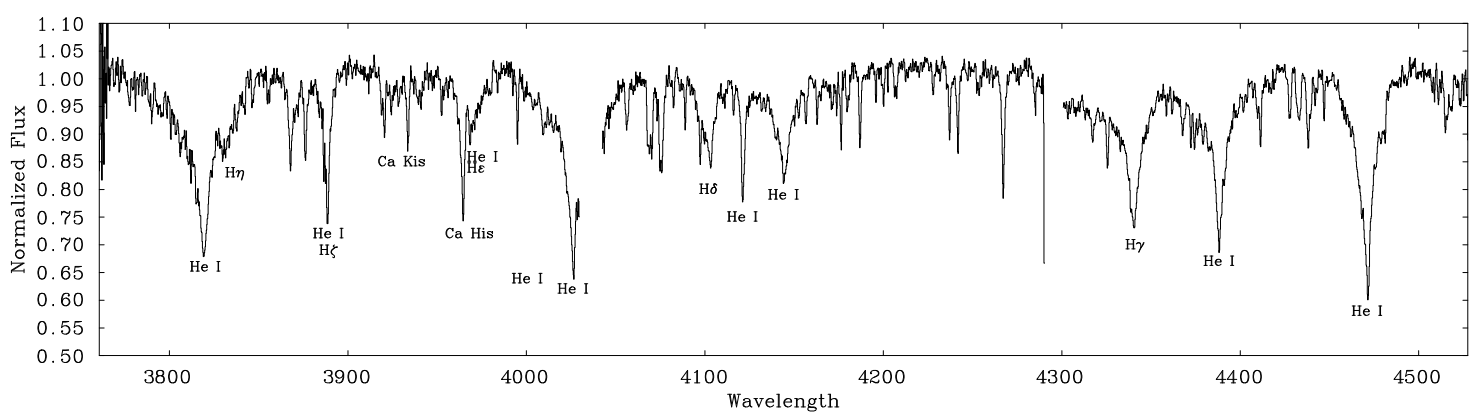

Figure 1: Coadded SALT RSS spectrum of the intermediate helium-rich subdwarf HE 0111-1526.

From previous observations, the He-rich sdOB star HE 0111-1526 had been identified as a potential binary. Follow-up RSS observations were obtained in 2012/13, giving radial-velocity measurements with a full amplitude of $40 \mathrm{~km} \mathrm{~s}^{-1}$ and a mean error of $\approx 5 \mathrm{~km} \mathrm{~s}^{-1}$. Owing to the distribution of eight observations over some 10 months, it has proved impossible to deduce a unique period, although the data suggest it should be no longer than a few days.

Ströer et al. [17] reported the extreme He-sdO star HE 0301-3039 to be a double He-sdO on the basis of two VLT-UVES spectra obtained as part of the Supernova Progenitor Survey [18]. Attempts to obtain repeat observations at sufficiently high-resolution and signal-to-noise with SALTRSS in 2011/12 have so far proved unsuccessful. Again, RSS throughput at the time of observation was lower than expected.

\section{Extreme helium stars}

During 2015, we have embarked on an ambitious programme to use SALT-HRS to carry out a high-time and high-spectral resolution study of the helium star BX Cir, which has a pulsation period of 2.5 hours [19], very similar to that of V652 Her [20]. Recent observations of the latter have demonstrated the presence of a shock at minimum radius, and differential expansion and contraction of the observable photosphere around the pulsation cycle [21]. Although the pulsation cycle is long compared with most SALT observing windows, BX Cir has a declination of $-66^{\circ}$, where SALT has a single continuous visibility window lasting over six hours. Observations with the SALT High Resolution Spectrograph (HRS) for the 2015 season have concluded, with $24 \times 1 \mathrm{~h}$ blocks completed. The data comprise some 275 individual spectra with excellent phase distribution. Reduction and analysis are in progress.

\section{Hydrogen-deficient binaries}

One of the first hydrogen-deficient stars to be discovered was the bright A-type supergiant $v$ Sgr, which has an orbital period of $120 \mathrm{~d}$ [22]. Approximately five other hydrogen-deficient 
binaries are known. Only one, KS Per, has a well-established period (360 d) [23, 24]. V1037 Sco has a reported period of $52.1 \mathrm{~d}$, and an eccentric orbit [25]. The orbital periods of V426 Car and BI Lyn are unknown. DY Cen, previously reputed to be a hot RCrB star, is apparently a carbon-rich hydrogen-deficient binary in a highly eccentric orbit of period 39.7d [26]. Only in the case of BI Lyn is the secondary star identifiable in the visible spectrum. The surface of DY Cen is heating at a rate of $250 \mathrm{~K} \mathrm{y}^{-1}$. Most of these hydrogen-deficient binaries show pulsations and variable emission, the latter uncorrelated (so far) with either the orbital period or the pulsations. Mass estimates suggest some may be potential SN Ib progenitors [27, 28].

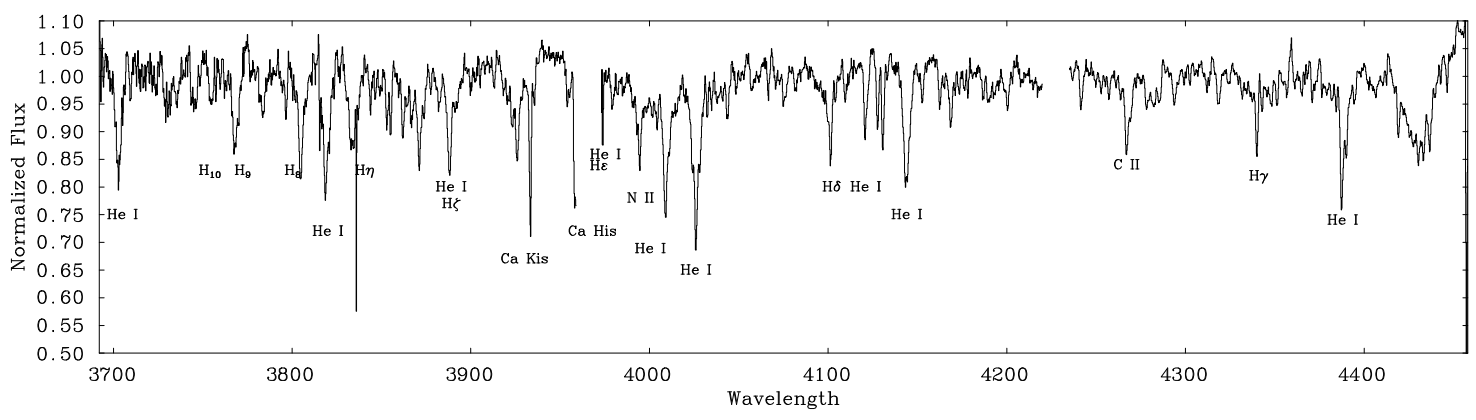

Figure 2: Coadded SALT RSS spectrum of the hydrogen-deficient binary V1037 Sco.

SALT-RSS and SALT-HRS observations have been attempted to address various questions regarding the orbital periods and related emission of V1037 Sco, V426 Car and DY Cen. At the time of writing, the HRS data have still to be reduced. The RSS velocities for DY Cen and V1037 Sco have proved impossible to interpret. A major problem encounterd in these early observations, but only realised during subsequent data analysis, is that the RSS calibration lamp has insufficient well-exposed lines in the blue to regularly allow a secure wavelength calibration.

\section{Conclusion}

SALT has offered the potential to make significant progress in understanding the late stages of stellar evolution from synoptic studies of pulsating and binary stars. As a consequence of lower than expected efficiency in RSS, a sub-optimal distribution of observing windows, and poor calibration images, many of those goals await realisation. The HRS data look promising, but substantial work has still to be done on the data-reduction side. It is imperative that SALT invest resources in developing robust data-reduction pipelines in order to exploit its full potential.

\section{Acknowledgments}

Thanks are due to the UK Salt Consortium, who enabled my attendance at the SALT Science Conference 2015, to Amy Holden who carried out the first analysis of the pulsating sdB star spectroscopy, to Pamela Martin for reprocessing much of the RSS spectroscopy, to Steve Crawford for generous advice, and to the Northern Ireland Dept for Culture Arts and Leisure for funding. 


\section{References}

[1] U. Heber, The atmosphere of subluminous B stars. II - Analysis of 10 helium poor subdwarfs and the birthrate of sdB stars, A\&A 155 (Jan., 1986) 33-45.

[2] Z. Han, P. Podsiadlowski, P. F. L. Maxted, T. R. Marsh, and N. Ivanova, The origin of subdwarf B stars - I. The formation channels, MNRAS 336 (Oct., 2002) 449-466, [astro-ph/o].

[3] P. Bergeron, F. Wesemael, G. Michaud, and G. Fontaine, Studies of hot B subdwarfs. VI Detailed calculations of radiative forces on metals in the envelopes and atmospheres of hydrogen-rich subdwarfs, ApJ 332 (Sept., 1988) 964-983.

[4] S. Geier, Hot subdwarf stars in close-up view. III. Metal abundances of subdwarf B stars, aa 549 (Jan., 2013) A110, [arxiv: 1211.2689].

[5] D. Kilkenny, C. Koen, D. O'Donoghue, and R. S. Stobie, A new class of rapidly pulsating star - I. EC 14026-2647, the class prototype, MNRAS 285 (Mar., 1997) 640-644.

[6] E. M. Green, G. Fontaine, M. D. Reed, K. Callerame, I. R. Seitenzahl, B. A. White, E. A. Hyde, R. Østensen, O. Cordes, P. Brassard, S. Falter, E. J. Jeffery, S. Dreizler, S. L. Schuh, M. Giovanni, H. Edelmann, J. Rigby, and A. Bronowska, Discovery of A New Class of Pulsating Stars: Gravity-Mode Pulsators among Subdwarf B Stars, ApJ 583 (Jan., 2003) L31-L34.

[7] S. Charpinet, G. Fontaine, P. Brassard, E. M. Green, and P. Chayer, Structural parameters of the hot pulsating B subdwarf PG 1219+534 from asteroseismology, A\&A 437 (July, 2005) 575-597, [astro-ph/0].

[8] D. O’Donoghue, A. E. Lynas-Gray, D. Kilkenny, R. S. Stobie, and C. Koen, A new class of rapidly pulsating star - IV. Oscillations in EC 20117-4014 and atmospheric analyses, MNRAS 285 (Mar., 1997) 657-672.

[9] R. Silvotti, R. Østensen, U. Heber, J.-E. Solheim, S. Dreizler, and M. Altmann, $P G$ 1325+101 and PG 2303+019: Two new large amplitude subdwarf B pulsators, A\&A 383 (Jan., 2002) 239-243.

[10] R. Oreiro, R. H. Østensen, E. M. Green, and S. Geier, 2M0415+0154: a new bright, short-period subdwarf B pulsator, A\&A 496 (Mar., 2009) 827-832.

[11] X. Zhang and C. S. Jeffery, Evolutionary models for double helium white dwarf mergers and the formation of helium-rich hot subdwarfs, MNRAS 419 (Jan., 2012) 452-464.

[12] H. T. Sener and C. S. Jeffery, Spectroscopic orbital elements for the helium-rich subdwarf binary PG 1544+488, Monthly Notices of the Royal Astronomical Society 440 (2014), no. 3 2676-2683. 
[13] S. Justham, P. Podsiadlowski, and Z. Han, On the formation of single and binary helium-rich subdwarf O stars, MNRAS 410 (Jan., 2011) 984-993, [arXiv: 1008. 1584].

[14] N. Naslim, C. S. Jeffery, A. Hibbert, and N. T. Behara, Discovery of extremely lead-rich subdwarfs: does heavy metal signal the formation of subdwarf B stars?, MNRAS 434 (Sept., 2013) 1920-1929, [arXiv:1407.7668]].

[15] N. Naslim, S. Geier, C. S. Jeffery, N. T. Behara, V. M. Woolf, and L. Classen, The helium-rich subdwarf CPD-20 1123: a post-common-envelope binary evolving on to the extended horizontal branch, MNRAS 423 (July, 2012) 3031-3038, [arXiv:1204.4387].

[16] H. Edelmann, Spectroscopic analyses of subluminous B stars: observational constraints for the theory of stellar evolutio, pulsation and diffusion. $\mathrm{PhD}$ thesis,

Friedrich-Alexander-Universität Erlangen-Nürnberg, 2003.

[17] A. Ströer, U. Heber, T. Lisker, R. Napiwotzki, S. Dreizler, N. Christlieb, and D. Reimers, Hot subdwarfs from the ESO supernova Ia progenitor survey. II. Atmospheric parameters of subdwarf O stars, A\&A 462 (Jan., 2007) 269-280, [astro-ph/0].

[18] R. Napiwotzki, N. Christlieb, H. Drechsel, H.-J. Hagen, U. Heber, D. Homeier, C. Karl, D. Koester, B. Leibundgut, T. R. Marsh, S. Moehler, G. Nelemans, E.-M. Pauli, D. Reimers, A. Renzini, and L. Yungelson, Search for progenitors of supernovae type Ia with SPY, Astronomische Nachrichten 322 (Dec., 2001) 411-418, [astro-ph/0].

[19] D. Kilkenny and C. Koen, The detection of small-amplitude variations in the extreme helium star LSS 3184, MNRAS 275 (July, 1995) 327-330.

[20] A. U. Landolt, On the optical variability of the helium stars HD 160641 and BD +13 3224., ApJ 196 (Mar., 1975) 789-790.

[21] C. S. Jeffery, D. Kurtz, H. Shibahashi, R. L. C. Starling, V. Elkin, P. Montañés-Rodríguez, and J. McCormac, Subaru and Swift observations of V652 Herculis: resolving the photospheric pulsation, MNRAS 447 (Mar., 2015) 2836-2851, [arXiv:1412.8672].

[22] R. E. Wilson, The orbit of the spectroscopic binary upsilon Sagittarii., Lick Observatory Bulletin 8 (1915) 132-133.

[23] W. P. Bidelman, The Peculiar Star HD 30353., ApJ 111 (Mar., 1950) 333.

[24] J. F. Heard and O. Boshko, The orbital elements of the hydrogen-poor spectroscopic binary HD 30353., AJ 60 (June, 1955) 162.

[25] D. J. Frame, P. L. Cottrell, A. C. Gilmore, P. M. Kilmartin, and W. A. Lawson, Hydrogen Deficient Binaries - Photometry and Orbits, MNRAS 276 (Sept., 1995) 383.

[26] N. Kameswara Rao, D. L. Lambert, D. A. García-Hernández, C. S. Jeffery, V. M. Woolf, and B. McArthur, The Hot R Coronae Borealis Star DY Centauri is a Binary, ApJ 760 (Nov., 2012) L3, [arXiv: 1210.4199]. 
[27] A. Uomoto, What stars become peculiar type I supernovae?, ApJ 310 (Nov., 1986) L35-L37.

[28] R. E. Dudley and C. S. Jeffery, The Mass Ratio of Upsilon-Sagittarii from Ultraviolet Radial Velocities, MNRAS 247 (Dec., 1990) 400. 